Sapouna et al, composite MREs

\title{
Dynamic mechanical properties of isotropic/anisotropic silicon magnetorheological elastomer composites
}

\author{
K Sapouna, Y P Xiong and R A Shenoi \\ Faculty of Engineering and the Environment, Fluid Structure Interactions Research Group, University \\ of Southampton, Boldrewood Campus, Southampton SO16 7QF, UK \\ E-mail: ks9g09@ @oton.ac.uk
}

\begin{abstract}
This study examines the principle of combining isotropic and anisotropic MREs in parallel and series configurations, to adjust the zero-field dynamic stiffness and damping capability of silicon MREs without compromising MR effect. The dynamic mechanical properties can be further tailored by adjusting the isotropic/anisotropic ratio. Damping of parallel configuration isotropic/anisotropic composites can be increased by combining MREs made with iron particles of small (4-6 $\mu \mathrm{m})$ and large $(<220 \mu \mathrm{m})$ diameter. In addition, we tested a novel anisotropic elastomer that combines two anisotropic MREs, with their particles aligned in different directions to achieve similar dynamic stiffness, damping and magnetorheological effect in those directions. The magnetorheological effect of the novel anisotropic/anisotropic composite MRE is $10 \%$ higher than that of pure anisotropic MRE. The axial, longitudinal and transverse dynamic mechanical properties, magnetorheological effect and the magnetic field-strain amplitude coupling effects were examined under a dynamic compressive strain where the amplitude was varied from $0.25 \%$ to $1.5 \%$.
\end{abstract}

Keywords: Magnetorheological elastomers, Dynamic stiffness, Composite, Magnetorheological effect

\section{Introduction}

Magnetorheological elastomers (MRE) are smart materials that consist of ferromagnetic particles suspended usually in a soft low permeability elastomer matrix like silicon rubber, natural rubber (NR) 
Sapouna et al, composite MREs

and Polyurethane rubber (PU). When the mixture is cured under the presence of a strong magnetic field the particles tend to place themselves along the direction of the magnetic field and form threedimensional chain structures inside the matrix. The produced MREs are called anisotropic or aligned. When the mixture is cured without the presence of a magnetic field, the filler particles are dispensed randomly inside the matrix and the elastomer is called isotropic. However, even isotropic MREs become anisotropic materials when the external field is applied. Thus, the terms isotropic and anisotropic MRE are conventional terms used in the literature [1], [2] to distinguish the two types of MR elastomers.

The performance of MR materials is usually assessed by a measure called magnetorheological (MR) effect which is the relative change of the modulus of elasticity when the field increases from OT to a certain value. Anisotropic silicon MREs are stiffer, have higher damping and higher MR effect than the equivalent isotropic MREs [3], [4]. The original zero field dynamic properties and maximum possible MR effect of any MRE depend on matrix elastomer, size, type and concentration of filler particles as well as strength of the magnetic field during curing in case of anisotropic MREs.

For common passive rubber isolators used in the industry, the designer selects the type, size and carbon black content of the rubber to adjust stiffness and damping to the desired levels while static load and vibration modes define if the device will operate in shear, compression or torsion mode. For active or semi active MRE isolators the situation is much different and the MR effect becomes the most important parameter. MREs with soft matrix elastomers like silicon and PU have a higher MR effect than the ones made of traditional rubbers used in vibration isolators like NR [4]- [7]. However, the low zero field stiffness and damping of silicon MREs limit their load carrying capability and isolation efficiency at high frequencies when used in vibration isolators.

In general, zero field dynamic stiffness and damping of MREs can be adjusted by increasing particle concentration [8]- [10] and for anisotropic MREs by increasing the magnetic field while curing [4]. However, it has been well established that the maximum MR effect in silicon MREs is achieved when the particle/matrix material ratio is about $30 \%$ per volume or $70 \%$ per weight [4], [11], [3] and for anisotropic MREs when the magnetic field while curing is close to the saturation level [12]. Thus, the optimum particle concentration for silicon MREs is fixed and the zero-field dynamic stiffness and damping cannot be increased after a point without compromising the MR effect.

It has been reported that the MR effect of isotropic and anisotropic silicon MREs is higher under compression than shear loading conditions [3], [8], [11], [13] and thus, MRE isolators working in pure compression mode become of interest. In seismic isolators working, static compression stiffness can be increased by using MRE-metal laminated structures [14] that need greater and heavier magnetic circuits. For most applications size and weight of the isolator are limiting factors. Thus, the gap in magnetic circuit of the isolator where the MRE is placed should be as small as possible to achieve high magnetic flux values. Decreasing the stiffness of the isolator by simply making the rubber thicker is not an option for MREs. 
Sapouna et al, composite MREs

Another way to improve the isolation characteristics of isolators with high damping rubbers at high frequencies, is to place a second rubber with lower damping factor and stiffness in parallel [15]. Under the same logic, combining isotropic and anisotropic MREs in one composite elastomer could produce a new MRE with lower zero field stiffness but similar damping capability than the anisotropic MRE itself without compromising MR effect. To the authors knowledge, this approach has never been examined before for MREs. In the published literature only Allajyarov et al [16] considered the theoretical magnetomechanical coupling of two magnetic elastomers into a bilayered composite material, to conclude that the composite elastomer responds better to the external field than a single component elastomer. However, they did not examine the case of placing the two elastomers in parallel or validated their results with experimental data.

In this experimental study, we check the above hypothesis by examining for the first time the dynamic compression properties of isotropic/anisotropic composite silicon MREs in parallel and series configurations (Figure 1) under a dynamic compressive strain with varying amplitude (from $0.25 \%$ to $1.5 \%$ ) at zero and at $0.5 \mathrm{~T}$ magnetic field. Only the magnetic field-strain amplitude coupling effect was considered in this study since the excitation frequency does not influence the MR effect [17], [18]. Furthermore, we show that the damping capability of the parallel combination composite MRE could be increased by combining MREs with iron particles of different sizes. For this reason, we tested first disk shaped composite isotropic-anisotropic MREs made with iron particles of small (4-6 $\mu \mathrm{m})$ and large $(<220 \mu \mathrm{m})$ diameter and compared them with the equivalent pure isotropic and anisotropic MREs.

In addition, we take this study one step further by examining the axial, transverse and longitudinal dynamic complex compression stiffness of cube shaped isotropic-anisotropic and anisotropicanisotropic (with particles aligned in different directions) composite MREs. The latter composite combination results to a novel anisotropic elastomer with similar zero field dynamic compression stiffness and MR effect in two directions, that is greater than the MR effect of pure MREs. This new type of anisotropic MRE could be used in applications where the MRE isolator is required to have equal stiffness and damping in two directions.

\section{Background theory}

When a sinusoidal compression strain is applied to an elastomer the material does not respond instantaneously but the resulting stress will be lagging the input by an angle $\delta$ called the loss angle. To represent this situation mathematically the modulus of elasticity $\mathrm{E}^{*}$ and dynamic stiffness $\mathrm{K}^{*}$ are considered as complex numbers of a real elastic element $\left(\mathrm{E}^{\prime}=\right.$ Storage modulus, $\mathrm{K}^{\prime}=$ real part of dynamic stiffness) and an imaginary viscous element $\left(\mathrm{E}^{\prime \prime}=\right.$ Loss modulus, $\mathrm{K}^{\prime \prime}=$ imaginary part of dynamic stiffness) $E^{*}=E^{\prime}+j E^{\prime \prime}$ and $K^{*}=K^{\prime}+j K^{\prime \prime}$ respectively. The absolute compression modulus $\left|E^{*}\right|$, absolute dynamic stiffness $\left|\mathrm{K}^{*}\right|$ and tangent of the loss angle tan $\delta$ are defined accordingly as:

$$
\left|\mathrm{E}^{*}\right|=\sqrt{\mathrm{E}^{\prime 2}+E^{\prime \prime 2}}
$$


Sapouna et al, composite MREs

$$
\begin{aligned}
& \left|\mathrm{K}^{*}\right|=\sqrt{\mathrm{K}^{\prime 2}+K^{\prime \prime 2}} \\
& \tan \delta=\frac{E^{\prime \prime}}{E^{\prime}}=\frac{K^{\prime \prime}}{K^{\prime}}
\end{aligned}
$$

When a compressive stress $\sigma$ is applied on a parallel configuration composite elastomer, it will be distributed across the cross-sectional area $A_{1}$ and $A_{2}$ of each individual rubber. In a similar way, when a stress $\sigma$ is applied on the series configuration composite elastomer the resulting strain $\varepsilon$ will be the sum of the individual strains $\varepsilon_{1}, \varepsilon_{2}$ of each rubber component, as illustrated in figure 1 .
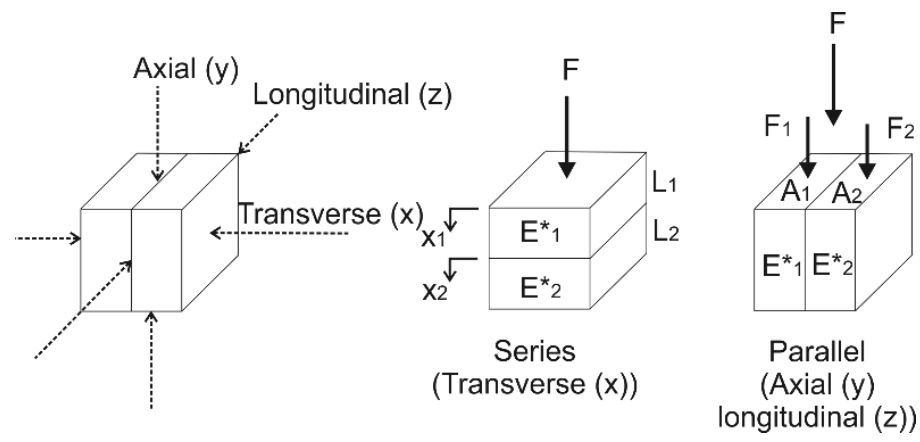

Figure 1: Definition of Series and parallel configuration composite elastomers

Setting $E_{1}{ }^{*}=E^{\prime}{ }_{1}+j E^{\prime \prime}{ }_{1}$ and $E_{2}{ }^{*}=E^{\prime}{ }_{2}+j E^{\prime \prime}{ }_{2}$ as the complex moduli of elasticity of the low $\tan \delta$ and the high $\tan \delta$ elastomer respectively, storage modulus $E^{\prime}$ and loss modulus $E^{\prime \prime}$ of the parallel configuration composite MRE are given by equations (4) and (5) [15], [19].

$$
\begin{aligned}
& E^{\prime}=\frac{E_{1}^{\prime}+a E_{2}^{\prime}}{1+a} \\
& E^{\prime \prime}=\frac{E^{\prime \prime}{ }_{1}+a E^{\prime \prime}{ }_{2}}{1+a}
\end{aligned}
$$

Where $a=\frac{A_{2}}{A_{1}}$ is the area ratio, $A_{1}$ is the area of the low tangent of the loss angle $\tan \delta$ rubber and $A_{2}$ is the area of high tangent of the loss angle tan $\delta$ rubber. Storage modulus $E^{\prime}$ and loss modulus $E^{\prime \prime}$ of the series configuration elestomer are given by equations (6) and (7) [15], [19].

$$
\begin{aligned}
E^{\prime} & =\frac{(1+b)}{\left(b E_{2}^{\prime}+E_{1}^{\prime}\right)} \frac{R c-d}{1+R^{2}} \\
E^{\prime \prime} & =\frac{(1+b)}{\left(b E_{2}^{\prime}+b E_{1}^{\prime}\right)} \frac{R d+c}{1+R^{2}}
\end{aligned}
$$

Where $b=\frac{l 1}{l 2}$ is the length ratio, $L_{1}$ is the legnth of the low tangent of the loss angle rubber and $L_{2}$ is the length of high tangent of the loss angle rubber and $R=\frac{E^{\prime \prime}{ }_{1}+b E^{\prime \prime}}{E_{2}{ }_{1}+b E^{\prime}{ }_{2}}, c={E^{\prime}}_{1} E^{\prime \prime}{ }_{2}+E^{\prime \prime}{ }_{1} E_{2}^{\prime}$ and $d=E^{\prime \prime}{ }_{1} E^{\prime \prime}{ }_{2}-E^{\prime}{ }_{1} E^{\prime}{ }_{2}$.

\section{Methodology}


Sapouna et al, composite MREs

\subsection{Series and parallel configuration isotropic/anisotropic composite MREs}

According to theory, the parallel or series configuration isotropic/anisotropic composite MR elastomer (Figure 2) could have a lower zero field dynamic compression stiffness than anisotropic MRE and higher zero field tangent of the loss angle than isotropic MRE. The exact zero field mechanical properties of the composite MRE could be adjusted by selecting appropriate area or length ratios and by combining MREs with different particle sizes.

To validate this theoretical assumption, we first tested two composite MRE samples that combined equal parts $(\alpha=\mathrm{b}=1)$ of isotropic and anisotropic elastomers in parallel and series configurations (Figure 2 ) and compared them to the pure isotropic and anisotropic MREs. The above samples were made with iron particles of average diameter of 4-6 $\mu \mathrm{m}$ that are mostly used in the literature and will be further refereed as small particles. In this case, area ' $\alpha$ ' and height ' $b$ ' ratios are defined as:

$$
\begin{aligned}
\alpha & =\frac{\text { Area of anisotrpic } M R E}{\text { Area of isotropic } M R E} \\
b & =\frac{\text { Height of anisotrpic } M R E}{\text { Height of isotropic } M R E}
\end{aligned}
$$

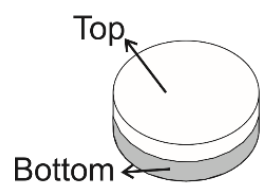

(a)

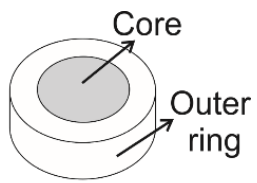

(b)

Figure 2: a) series configuration and b) parallel configuration composite disk MRE samples.

Then, three additional parallel configuration composite MRE samples with area ratio ' $\alpha$ ' of $1 / 3,2 / 3$ and 3 respectively were made, using the above mentioned small particles (4-6 $\mu \mathrm{m}$ diameter) and much larger particles with a range of diameters lower than $220 \mu \mathrm{m}$ further refereed as large particles. This set of samples were disk shaped as indicated in BS ISO 4664-1:2011 standard on determination of dynamic properties of rubber for compression tests and they are described in detail in Table 2 and Figure 3 . The mechanical properties of these composite MREs were compared to the pure isotropic and anisotropic MREs with small and large particles mentioned in Table 1.

Table 1: Pure disk MRE samples.

\begin{tabular}{ccc}
\hline Name & Type & Particles \\
\hline Is-1 & Isotropic & Large $(<220 \mu \mathrm{m})$ \\
Is-s & Isotropic & Small $(4-6 \mu \mathrm{m})$ \\
An-1 & Anisotropic & Large $(<220 \mu \mathrm{m})$ \\
An-s & Anisotropic & Small $(4-6 \mu \mathrm{m})$ \\
\hline
\end{tabular}


Sapouna et al, composite MREs

Table 2: Composite disk MRE samples.

\begin{tabular}{ccccc}
\hline Name & $a$ & MRE type & Particles & Part \\
\hline Pa-1 & $1 / 3$ & Anisotropic & large & Core \\
& & Isotropic & large & Out. ring \\
& & Anisotropic & large & Core \\
Pa-2 & $2 / 3$ & Anison & small & Out. ring \\
& & Isotropic & large & Core \\
Pa-3 & 3 & Isotropic & large & Out. ring \\
& & Anisotropic & Core \\
Pa-4 & 1 & Anisotropic & Small & Cong \\
& & Isotropic & Small & Out. ring \\
& & & & \\
\hline Se-1 & 1 & Anisotropic & Small & Upper \\
& & Isotropic & Small & Lower \\
\hline
\end{tabular}
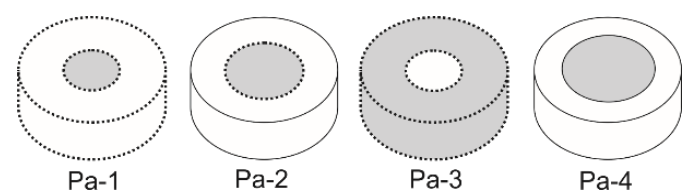

Figure 3: Parallel configuration isotropic (white) /anisotropic (grey) composite disk samples with small (solid line) and large (dashed line) particles.

\subsection{Dynamic mechanical properties of composite MREs in three directions}

In practical vibration isolation systems where the used elastomer is subjected to multidirectional loads, the dynamic stiffness of the elastomer in each direction must be defined. This is a straightforward process for isotropic materials like isotropic MREs at zero field, but not for anisotropic and composite MREs. The pre-aligned particle chains, in anisotropic MREs, result to a highly anisotropic material with a much higher zero field dynamic stiffness and tangent of the loss angle in one direction. However, combining two anisotropic MREs, with their particles aligned in different directions, can produce an anisotropic elastomer with similar stiffness and damping in those directions.

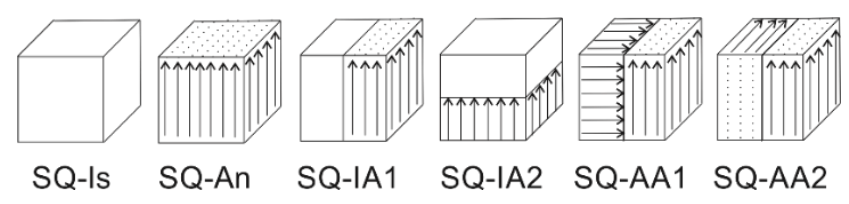


Sapouna et al, composite MREs

Figure 4: Cube samples combining isotropic and anisotropic MREs. The blank blocks represent isotropic MREs while the arrows in the boxes represent the direction of the aligned chains in anisotropic MREs.

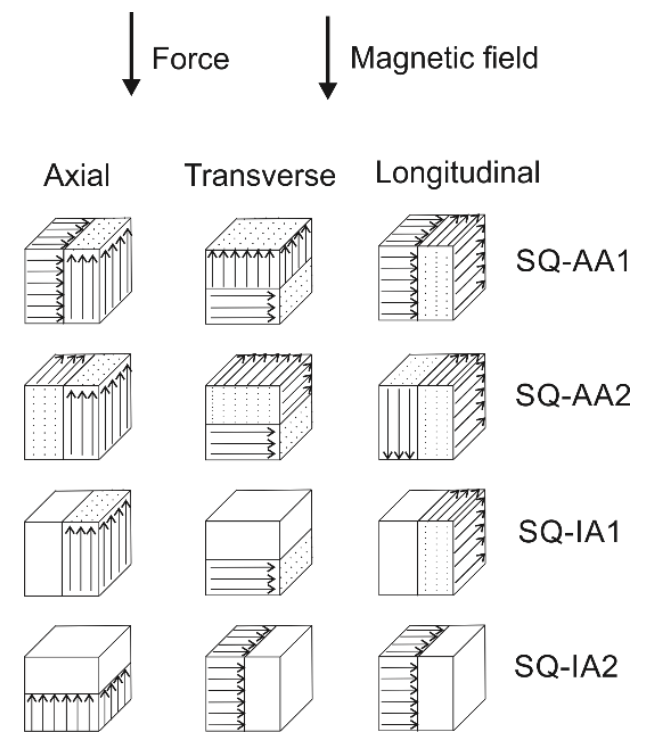

Figure 5: Defining axial, transverse and longitudinal mechanical characteristics of composite MREs.

To examine the dynamic compression stiffness and damping of composite MRE samples in all three directions, we used cube samples of pure isotropic and anisotropic elastomers as well as all their parallel and series combinations (sample SQ-Is, SQ-An, SQ -IA1 and SQ-IA2). In addition, cube samples SQAA1 and SQ-AA2 having two anisotropic halves with different particle chain directions were also examined. All cube samples are illustrated in Figure 4. The cube shape allows for the axial, transverse and longitudinal stiffness and damping of the composite materials to be defined by simply rotating the sample, as illustrated in Figure 5. The axial, transverse and longitudinal real dynamic stiffness K' and tangent of the loss angle tan $\delta$ of composite cube samples are defined accordingly.

\section{Details of experiment}

\subsection{MRE samples manufacture}

For our experiments, we manufactured silicon Rubber MREs using conventional spherical shape carbonyl iron particles with an average diameter of 4-6 $\mu \mathrm{m}$ (Sigma-Aldrich) and much larger particles with a range of diameters lower than $220 \mu \mathrm{m}$ (Sigma-Aldrich part No 12310). All samples were composed of $30 \%$ of volume carbonyl iron particles and $70 \%$ of volume two component silicon rubber (4664 Elastosil Wacker Silicones). The silicon rubber components were first mixed together and then the iron particles were added. The mixture was placed in a vacuum chamber for about 10 min to remove the air bubbles trapped inside during mixing and was finally poured in aluminium moulds leaving them to cure for 24 hours at room temperature. 
Sapouna et al, composite MREs

All disk samples were of $29 \pm 5 \mathrm{~mm}$ diameter and 12.5 \pm 5 height, according to BS ISO 4664:2011 standard. To make anisotropic MREs, the moulds were placed between two strong neodymium permanent magnets (diameter $=40 \mathrm{~mm}$ and thickness $=10 \mathrm{~mm}$ ) that produce a stable magnetic field of about 0.5 Tesla at a $12.5 \mathrm{~mm}$ air gap. The flux density was measured by placing a magnetic probe in the middle of the $12.5 \mathrm{~mm}$ gap using aluminium blocks on the sides to hold the permanent magnets apart.

The manufacturing process of the novel composite MR elastomers involves two stages. For all parallel combination samples (Figure 2 (b)), the anisotropic core cylinders were first made in the appropriate aluminium moulds following the same process described earlier. After they had cured, they were placed in the middle of another aluminium mould and the isotropic MRE was poured to fill the gaps and left to cure again at room temperature. By this way perfect adhesion between the two parts was achieved.

For the series combination sample Se_1, only one type of sample was made composed by an anisotropic with small particles top part and an isotropic with small particles bottom part. In this case an anisotropic cylinder of $12.5 \mathrm{~mm}$ height was first made, then it was cut in the middle and one half part was placed at the bottom of the aluminium mould. The isotropic part was then cured on top of it. The length of each individual part is $6.25 \mathrm{~mm}$, half of the $12.5 \mathrm{~mm}$ total MRE height.

The cube shaped MRE samples were manufactured with similar procedure. For the anisotropic parts, the moulds were placed between two strong neodymium permanent magnets (diameter $=40 \mathrm{~mm}$ and thickness $=10 \mathrm{~mm}$ ) that produce a stable magnetic field of about 0.35 Tesla at a $22 \mathrm{~mm}$ air gap. Samples SQ-IA1, SQIA2, SQ-AA1 and SQ-AA2 were manufactured by cutting a cube pure anisotropic sample in the middle, place one half in the bottom of the mould, rotate the mould and then cure the other half on top of it, to achieve perfect adhesion. By this way all anisotropic parts were cured under the same magnetic field of $0.35 \mathrm{~T}$.

\subsection{Test setup}

The dynamic compression tests were performed according to BS ISO 4664-1:2011 standard, using INSTRON PULS E1000 electromechanical dynamic tester and two custom made aluminium compression plates. The test system is illustrated in Figure 6. The samples where first preloaded under static load for four cycles to avoid the Mullins effect. One strain amplitude sweep cycle, from $0.25 \%$ to $1.5 \%$, was then performed at a constant frequency of $5 \mathrm{~Hz}$ and $10 \%$ static prestrain.

The magnetic field during testing was achieved again by placing the MRE samples between two strong neodymium permanent magnets of diameter $=40 \mathrm{~mm}$ and thickness $=10 \mathrm{~mm}$ each. These produced a stable magnetic field of $0.5 \mathrm{~T}$ (at a gap of $12.5 \mathrm{~mm}$ ) for cylinder samples and $0.35 \mathrm{~T}$ (at a gap of $22 \mathrm{~mm}$ ) for cube samples. Again, the flux density was measured by placing a magnetic probe in the middle of the gap using aluminium blocks on the sides to hold the permanent magnets apart. 
Sapouna et al, composite MREs

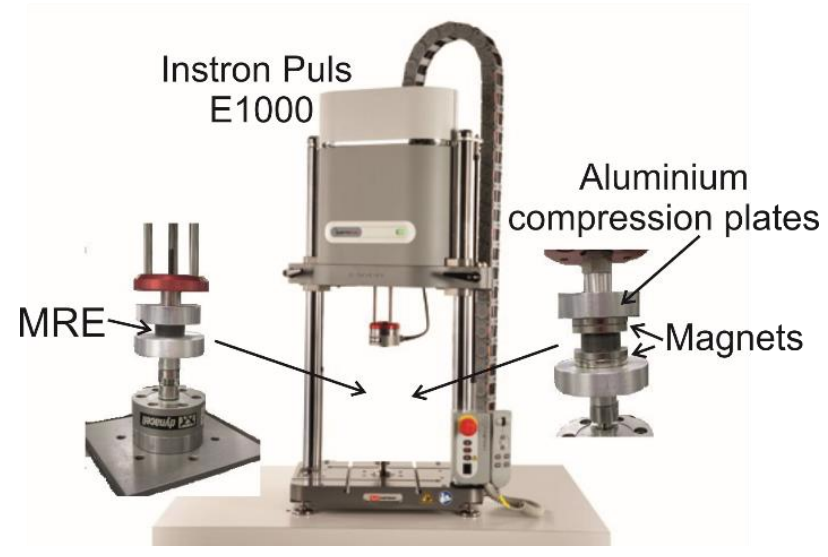

Figure 6: Experimental test setup.

In order to make safe conclusions when comparing the different types of MRE samples between each other, again the instructions of the ISO 4664-1:2011 standard were followed. Therefore, we manufactured five different batches of all pure and composite MRE samples and three batches were finally selected disregarding those with the highest and lowest MR effect. In this report the experimental values reported are the average of these three samples.

\section{Results and discussion}

\subsection{Pure isotropic and anisotropic MREs}

In this section, we examine the behaviour of pure isotropic and anisotropic MREs with large $(<220 \mu \mathrm{m})$ and small $(6 \mu \mathrm{m})$ particles. Figure 7 illustrates the zero-field storage E' modulus, loss E', modulus and tangent of the loss angle tan $\delta$ and the equivalent magnetorheological effect (at 0.5T) of all pure MREs in respect to strain amplitude. MRE samples with large particles have higher values of storage modulus E' thus, they are slightly stiffer than the samples with small particles. As strain amplitude increases absolute both moduli decrease, which is nothing more than the Payne effect observed for all filled rubbers. Payne effect is more pronounced in anisotropic MREs due to the destruction-deformation mechanism of the filler chain structure inside the matrix when the material is overstressed, that makes it softer [18].

Both isotropic MREs have similar tangent of the loss angle $\tan \delta$ (Figure 7c) and therefore, have similar damping capability that increases slightly with increasing strain amplitude. The results agree with the findings of other published studies [20]. On the other hand, anisotropic samples with large particles have much higher tangent of the loss angle tan $\delta$ than anisotropic samples with small particles that increases until $0.75 \%$ strain amplitude to decrease later. This behaviour can be better understood by observing how loss modulus E" (Figure 7b) varies with strain amplitude for all pure samples. Both anisotropic samples decrease their loss modulus E", with increasing strain amplitude but this drop is higher for anisotropic MREs with large particles because large filler particles tend to cause more destruction to the matrix-filler structure with increasing load. As a result, the material becomes softer 
Sapouna et al, composite MREs

and the particles can move easier inside the matrix. Thus, less energy is dissipated and damping capability is decreased.

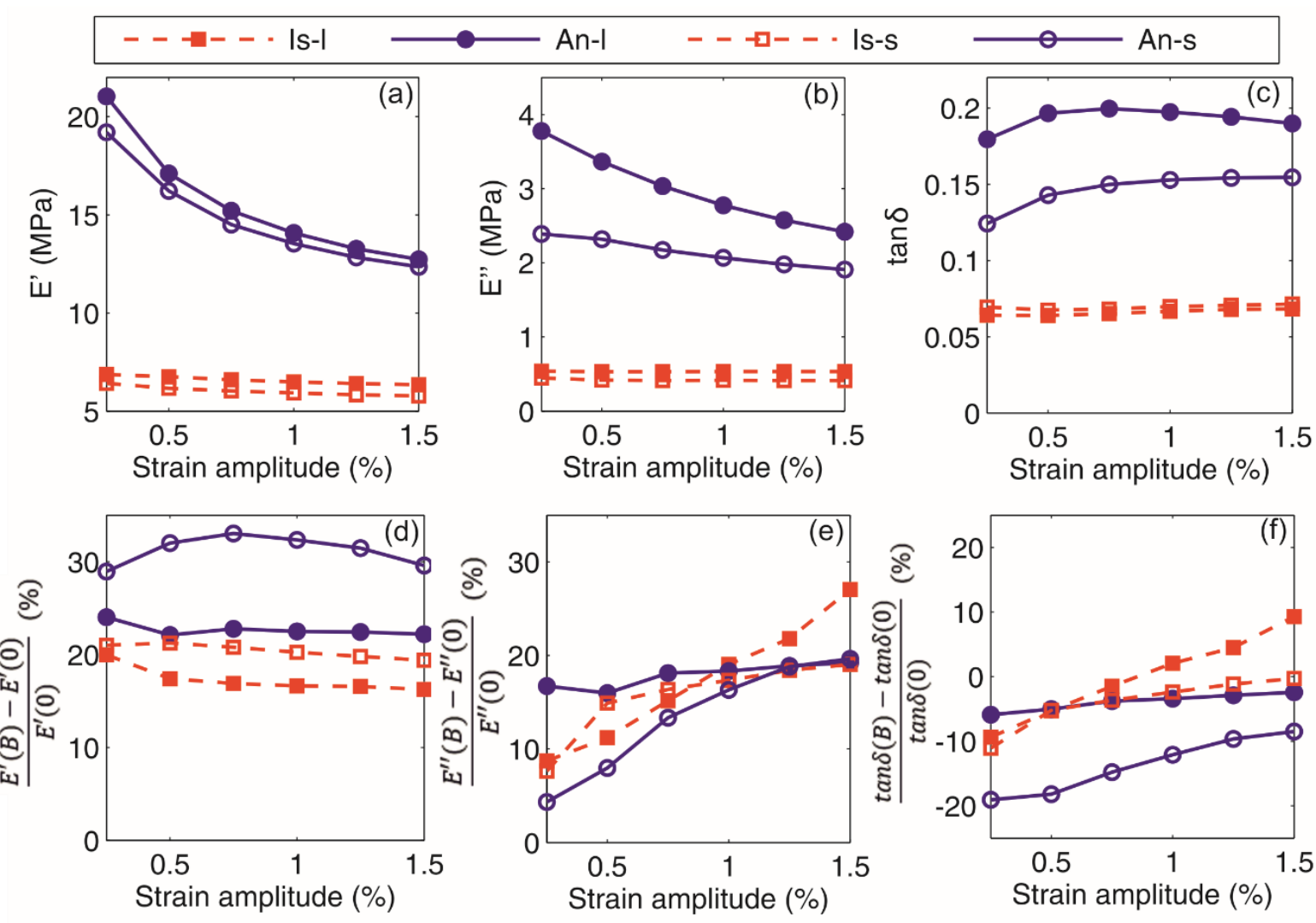

Figure 7: Zero-field values of a) storage E' modulus, b) loss E'" modulus, c) tangent of the loss angle $\tan \delta$ and MR effect of d) storage E' modulus, e) loss E' modulus, f) tan $\delta$ of isotropic and anisotropic MREs with large and small particles in respect to strain amplitude (measured at $5 \mathrm{~Hz}$ frequency and $10 \%$ prestrain).

All samples increase both their moduli of elasticity E' and E" (Figure 7d and 7e) when placed between the permanent magnets but at different rates. The relative increase is much higher for storage modulus E' than loss modulus E', and thus, more energy is stored than dissipated and the damping capability of the material decreases slightly with the application of the magnetic field. The storage modulus E' relative MR effect decreases slightly with increasing strain amplitude while loss modulus E" relative MR effect increases, again at different rates due to Payne effect. As strain amplitude increases, the destruction-deformation mechanism of the particles-matrix structure comes in place and the particles consume more energy to overcome the obstacles and align themselves parallel to the magnetic field. This also causes $\tan \delta$ MR effect (Figure 7f) to increase with increasing strain amplitude.

Anisotropic MREs have higher storage modulus E' relative MR effect than the equivalent isotropic samples, due to their original chain structure that allow the particles to move easier inside the matrix elastomer to align parallel to the direction of the magnetic field. In the case of isotropic MREs, the 
Sapouna et al, composite MREs

particles need to perform more complicated movements to align themselves that are limited by the matrix elasticity and the position of the neighbouring particles [18]. Therefore, more energy is needed for the particles to move, which explains the higher relative MR effect of loss modulus E" of isotropic samples compared to anisotropic MRE with small particles An-s.

The anisotropic sample with small particles An-s has a higher MR effect of storage modulus E' (Figure 7d) but a lower MR effect of loss modulus E'" (Figure 7e) than the one with large particles An1. This is attributed to bigger particles creating a more stable matrix-particle structure that resist the effect of the magnetic field because large particles require greater magnetic forces than smaller particles to move inside the matrix. For the same reason, the MR effect of loss modulus E"' and tan $\delta$ of sample An-l remains unaffected from strain amplitude, while in practice its damping capability is not influenced by the magnetic field in contrast to samples An-s and Is-1 where a strong MR effect of loss modulus E' '-strain amplitude coupling effect is observed (Figure 7e). However, the zero field values of loss modulus E' and $\tan \delta$ of isotropic samples are small and a $10 \%$ increase on their value could be close to measurement error. Therefore, we could assume that the damping capability of isotropic MREs is not greatly influenced by the magnetic field.

On another perspective, increasing particle size could lead to the formation of agglomerates at the bottom of the sample during curing due to gravitational forces [12]. Using smaller particles reduces gravitational sedimentation while anisotropic samples require lower magnetic field to create similar chain structures to samples with large particles.

\subsection{Series and parallel configuration composite MREs}

The zero-field storage E' and loss E' moduli of the composite MREs can be estimated by substituting $E^{\prime}{ }_{1}=6.75, E^{\prime \prime}{ }_{1}=0.43, E^{\prime}{ }_{2}=17.11$ and $E^{\prime \prime}{ }_{2}=3.36$ to equations (6) and (7) for samples Pa-1, Pa-3 where $\alpha=1 / 3$ and $\alpha=3$ respectively. $E^{\prime}{ }_{1}=6.16, E^{\prime \prime}{ }_{1}=0.416, E^{\prime}{ }_{2}=16.22$ and $E^{\prime \prime}{ }_{2}=2.32$ to equations (6) and (7) for samples Pa-4 and to equations (8) and (9) for sample se- 1 where $\alpha=b=1$. Finally, $E^{\prime}{ }_{1}=6.16, E^{\prime \prime}{ }_{1}=0.416, E^{\prime}{ }_{2}=17.11$ and $E^{\prime \prime}{ }_{2}=3.36$ to equations (6) and (7) for samples Pa-3 where $\alpha=2 / 3$. Absolute modulus $\left|E^{*}\right|$ and tangent of the loss angle $\tan \delta$ are then determined from equations (2) and (4). The calculated and measured values of all composite disk samples are presented in Table 3 (at $0.5 \%$ strain amplitude, $5 \mathrm{~Hz}$ loading frequency and $10 \%$ prestrain test conditions). The theoretical equations can therefore, provide a good estimation for the actual measured values.

Table 3: Zero field measured and calculated mechanical properties of composite disk MREs.

\begin{tabular}{ccccc}
\hline & \multicolumn{2}{c}{ Measured } & \multicolumn{2}{c}{ Calculated } \\
\cline { 2 - 5 } & $\left|E^{*}\right|$ & $\tan \delta$ & $\left|E^{*}\right|$ & $\tan \delta$ \\
& $(\mathrm{MPa})$ & & $(\mathrm{MPa})$ & \\
\cline { 2 - 5 } $\mathrm{Pa}-1$ & 9.1 & 0.12 & 9.5 & 0.13 \\
$\mathrm{~Pa}-2$ & 11.3 & 0.15 & 10.8 & 0.15 \\
\hline
\end{tabular}


Sapouna et al, composite MREs

\begin{tabular}{ccccc}
\hline Pa-3 & 14.8 & 0.19 & 14.96 & 0.17 \\
Pa-4 & 11.2 & 0.12 & 11.38 & 0.12 \\
Se-1 & 8.9 & 0.1 & 9.04 & 0.09 \\
\hline
\end{tabular}

The zero-field damping capability of a composite sample can be improved by using MREs with iron particles of different sizes. For example, consider samples $\mathrm{Pa}-2$ and $\mathrm{Pa}-4$ where the anisotropic to isotropic area ratio ' $\alpha$ ' of sample Pa-2 was selected so to have similar dynamic stiffness to sample Pa4 with unit area ratio. Using an anisotropic MRE core made of large particles to a cylinder isotropic MRE made of small particles (sample Pa-2), increases tangent of the loss angle for small strains by $25 \%$ while keeping stiffness similar to the case where the anisotropic core was made of small particles (sample Pa-4). Therefore, it is possible to tailor the dynamic mechanical properties of composite MREs by selecting appropriate MRE material, iron particle size and dimensions of each part.

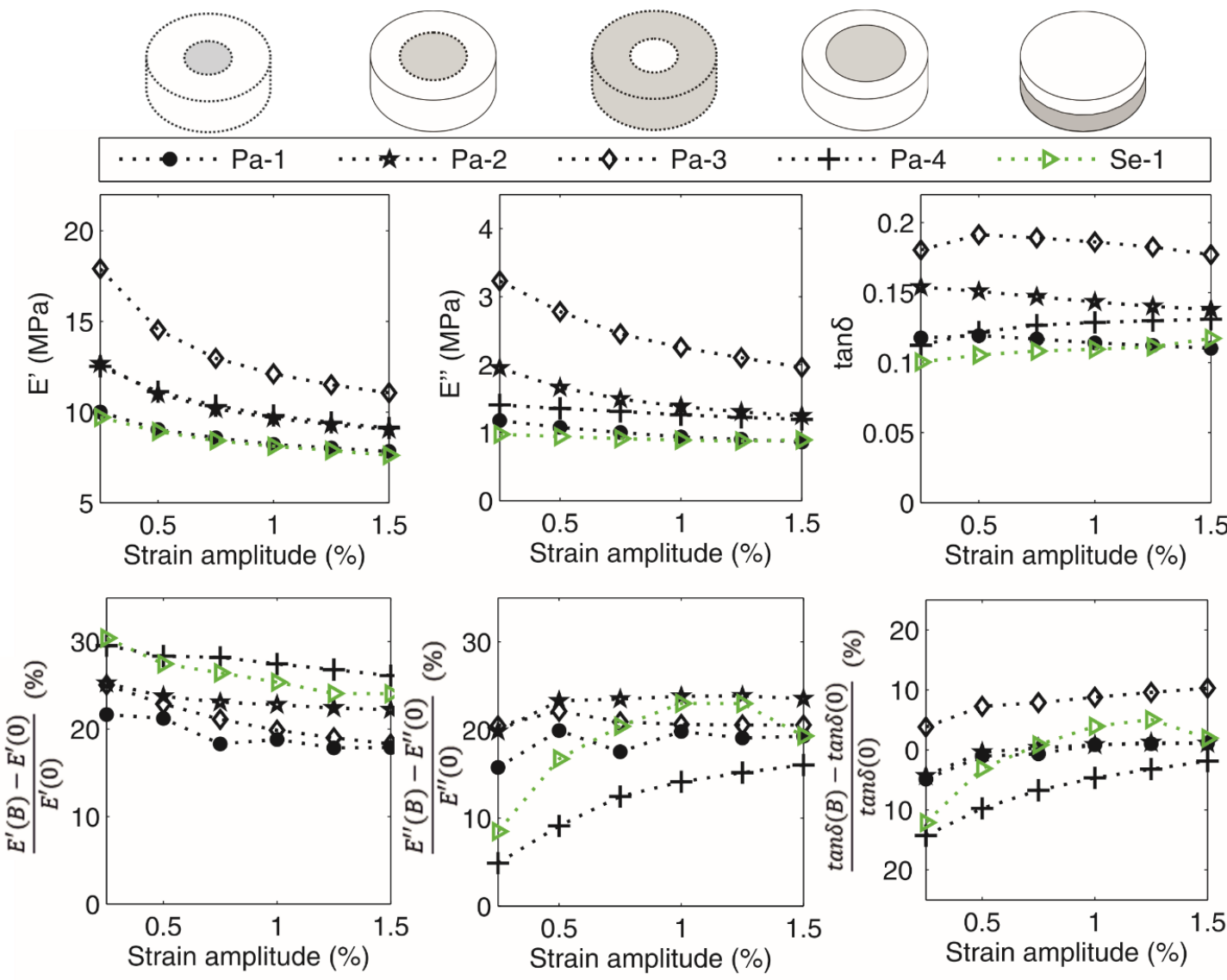

Figure 8: Zero-field values and MR effect of storage E' modulus, loss E' modulus and tangent of the loss angle tan $\delta$ of disk composite MREs in respect to strain amplitude (measured at $5 \mathrm{~Hz}$ frequency and $10 \%$ prestrain).

Figure 8 illustrate the storage E' modulus, loss E', modulus and tangent of the loss angle tan $\delta$ and the equivalent MR effect of all disk composite MREs in respect to strain amplitude. All composite samples drop their storage E' and loss modulus E"' with increasing strain amplitude but not as fast as 
Sapouna et al, composite MREs

pure anisotropic MREs due to the contribution of the isotropic part. As amplitude increases the matrixparticle structure is destroyed for both isotropic and anisotropic parts and the particles move inside the matrix causing both moduli of elasticity to decrease while this destruction-deformation mechanism is more pronounced in anisotropic MREs [18]. The behaviour of E' and E" at zero field for parallel composite samples with increasing amplitude is the simple sum of E' and E" of each isotropic and anisotropic part.

For example, the anisotropic to isotropic area ratios ' $\alpha$ ' of samples Pa-4 and Pa-2 were selected so that they have the same storage modulus E' but sample Pa-2 has higher loss modulus E'" that drops faster with increasing strain amplitude. This is because the large particles of the anisotropic core of sample Pa-2 create a greater destruction to the matrix-filler structure than the much smaller particles of the anisotropic core of sample Pa-4. As amplitude increases and the anisotropic material becomes softer, large particles of sample Pa-2 can move easier inside the matrix destroying their chain like structure and less energy is dissipated. Since the energy dissipated by the isotropic part is not influenced by strain amplitude both samples end up having the same loss modulus E" and $\tan \delta$ at $1.5 \%$ strain amplitude. Same principle applies for samples Se-1 and Pa-1.

When the magnetic field is applied the particles start moving inside the matrix to align parallel to the direction of the magnetic field which is easier for the anisotropic part. If a magnetic field was applied in the axial direction to an unconstrained composite MRE, it would elongate in the axial direction and get squeezed in the lateral direction [16] while, the elongation of the anisotropic part would be greater than that of the isotropic part. Since the magnetic forces between particles in the anisotropic part are higher than the isotropic part, there would be an interface layer between the two where the particles of the anisotropic MRE would attract particles of the isotropic one. The top surface of sample Pa-4 and side surface of sample Se-1 would then be curved. In this case, the composite MREs are loaded under a static and dynamic compression strain that restrict their movement. For parallel combination samples, the load is not evenly distributed and the anisotropic part supports most of the load.

Under a $0.5 \mathrm{~T}$ magnetic field, parallel combination sample Pa-4 increases its storage modulus by $28 \%$ while series combination sample Se-1 by $27 \%$. These values are very close to the $31 \% \mathrm{MR}$ effect of pure anisotropic An-s sample and higher than the 21\% MR effect of pure isotropic Is-s sample. When the field is applied the stiffness of the anisotropic part of samples Pa-4 and Se-1 increases $10 \%$ more than the one of the isotropic part. Since they are made of equal parts the total MR effect of the composite samples should be the sum of half the MR effects of each part (26\% for storage modulus). However, due to the presence of the interface layer between the two parts the actual MR effect is slightly higher. The loss modulus E" relative MR effect is $6 \%$ higher for sample Se-1 than sample Pa-4 because the interface layer is perpendicular to the applied load and magnetic field in contrast to sample $\mathrm{Pa}-4$ where it is parallel. Thus, the particles inside the interface layer of sample Se-1 cannot move inside the matrix as easy as in sample Pa-4 and need more energy to align with the magnetic field. 
Sapouna et al, composite MREs

Composite samples Pa-1 and Pa-3, that have both parts are with large particles show similar MR effect of storage modulus with the equivalent pure anisotropic MRE An-l. We assume that this is because large particles form greater magnetic forces between each other and thus, the interface layer between the two parts is wider increasing the total MR effect. Sample Pa-3 appears to be the only one to increase its $\tan \delta$ with the magnetic field by $5 \%$ that corresponds to an actual increase of about 0.01 that could be easily regarded as measurement error. The same applies to samples Pa-2 and Pa-1 and therefore, we can assume that tangent of loss factor $\tan \delta$ of samples made with large particles is not influenced by the magnetic field.

\subsection{Axial, transverse and longitudinal dynamic mechanical properties of composite MREs}

Since the cube MRE samples used for this type of test are not of the standard disk shape defined by ISO standard, the real part of dynamic stiffness K' will be reported instead of storage modulus E'. The axial, transverse and longitudinal dynamic absolute stiffness and tangent of the loss angle of all cube composite samples can be estimated by substituting $E_{1}^{\prime}=K^{\prime}{ }_{i s o} \frac{L_{1}}{A_{1}}, E^{\prime \prime}{ }_{1}=K^{\prime \prime}{ }_{i s o} \frac{L_{1}}{A_{1}}, E^{\prime}{ }_{2}=K^{\prime}{ }_{\text {an }} \frac{L_{2}}{A_{2}}$, $E^{\prime \prime}{ }_{2}=K^{\prime \prime}{ }_{a n} \frac{L_{2}}{A_{2}}$ and $a=b=1$ to equations (6), (7), (8) and (10). Where $K^{\prime}{ }_{\text {iso }}$ and $K^{\prime \prime}{ }_{\text {iso }}$ are the real and imaginary part of dynamic stiffness of the isotropic sample, while $K_{a n}^{\prime}$ and $K^{\prime \prime}$ an the real and imaginary part of dynamic stiffness of the anisotropic sample. For cube samples $\alpha=\mathrm{b}=1, L_{1}=L_{2}=L / 2$ and $A_{1}=A_{2}=A / 2$ where $\mathrm{L}$ length of cube $A=L * L$ area of each side. The two different MRE parts are combined in series or parallel configurations depending the direction of interest as defined in Figure 4 and Figure 5. The zero-field measured and calculated axial, transverse and longitudinal dynamic stiffness $\left|K^{*}\right|$ and tangent of the loss angle $\tan \delta$ of all cube samples, taking under consideration the particle alignment of the anisotropic parts, are shown in Table 4.

Table 4: Zero field measured and calculated $\left|K^{*}\right|$ and tan $\delta$ of cube MREs (at $0.5 \%$ amplitude, $5 \mathrm{~Hz}$ frequency and $10 \%$ prestrain).

\begin{tabular}{cccccc}
\hline \multirow{2}{*}{$\begin{array}{c}\text { MRE } \\
\text { sample }\end{array}$} & \multicolumn{2}{c}{ Measured } & \multicolumn{2}{c}{ Calculated } \\
\cline { 2 - 5 } & $\left|K^{*}\right|$ & $\tan \delta$ & $\left|K^{*}\right|$ & $\tan \delta$ & \\
\hline SQ-Is & 112 & 0.06 & \multicolumn{3}{c}{ Same for all directions } \\
SQ-An & 323 & 0.15 & & & Axial \\
& 122 & 0.07 & & & Transverse \\
& 122 & 0.07 & & & Longitudinal \\
SQ-AA1 & 213 & 0.13 & 223 & 0.13 & Axial \\
& 208 & 0.087 & 180 & 0.09 & Transverse \\
& 118 & 0.07 & 122 & 0.07 & Longitudinal
\end{tabular}


Sapouna et al, composite MREs

$\begin{array}{cccccc}\text { SQ-AA2 } & 224 & 0.134 & 223 & 0.13 & \text { Axial } \\ & 130 & 0.07 & 122 & 0.07 & \text { Transverse } \\ & 210 & 0.12 & 223 & 0.13 & \text { Longitudinal } \\ \text { SQ-IA1 } & 227 & 0.13 & 228 & 0.13 & \text { Axial } \\ & 122 & 0.06 & 118 & 0.06 & \text { Transverse } \\ & 115 & 0.06 & 117 & 0.06 & \text { Longitudinal } \\ \text { SQ-IA2 } & 191 & 0.08 & 170 & 0.08 & \text { Axial } \\ & 119 & 0.06 & 117 & 0.06 & \text { Transverse } \\ & 120 & 0.06 & 117 & 0.06 & \text { Longitudinal }\end{array}$

For example, for the axial stiffness of sample SQ-IA1 an isotropic part is considered in parallel to an anisotropic part with particles aligned in the same direction of the applied force. Axial stiffness of sample SQ-IA1 can therefore be estimated by using the stiffness of isotropic sample SQ-Is and the axial stiffness of anisotropic sample SQ-An (mentioned in Table 4), in equation (6) setting area ratio $\alpha=1$. However, for calculating the transverse stiffness the isotropic part is in series with the anisotropic part with particles aligned perpendicular to the applied force. In this case transverse stiffness of sample SQIA1 is estimated by using the stiffness of isotropic sample SQ-Is and the transverse stiffness of anisotropic sample SQ-An (mentioned in Table 4), in equation (8) setting length ratio $b=1$. In a similar way, the longitudinal stiffness of sample SQ-IA1 is estimated by using the stiffness of isotropic sample SQ-Is and the longitudinal stiffness of anisotropic sample SQ-An (mentioned in Table 4), in equation (6) setting area ratio $\alpha=1$.

When the particles of the anisotropic MREs are aligned perpendicular to the applied load (transverse and longitudinal), zero field stiffness K' and tangent of the loss angle tan $\delta$ are close to the isotropic SQIs sample. Under the same logic, the transverse and longitudinal stiffness and tangent of the loss angle of composite samples SQ-IA1 and SQ-IA2 are between the transverse stiffness of anisotropic SQ-An and isotropic SQ-Is MREs. Composite sample SQ-AA1 has similar axial and transverse stiffness K', but the transverse tangent of the loss angle $\tan \delta$ is higher since the two anisotropic parts are connected in parallel in the first case but in series in the second. However, sample SQ-AA2 has similar axial and longitudinal stiffness and tangent of the loss angle since the two anisotropic parts are connected in parallel in both cases. Longitudinal stiffness and tangent of the loss angle of sample SQ-AA1 as well as transverse stiffness and tangent of the loss angle of sample SQ-AA2 have similar values to the transverse anisotropic MREs.

The variation of zero field real dynamic stiffness $\mathrm{K}^{\prime}$ and tangent of the loss angle tan $\delta$ and their relative MR effect of isotropic, anisotropic, composite SQ-IA1 and composite SQ-IA2 MRE samples in respect to strain amplitude are illustrated in Figure 9. The longitudinal dynamic mechanical properties and MR effects of composite samples SQ-IA1 and SQ-IA2 are not illustrated because they are the same 
Sapouna et al, composite MREs

with the transverse ones for each sample. Payne effect is also observed here with the dynamic stiffness dropping with increasing strain amplitude while tangent of the loss angle increases slightly. In general, the same trends observed in the previous section regarding zero field dynamic stiffness $\mathrm{K}$ ' and $\tan \delta$ of composite samples also apply here.

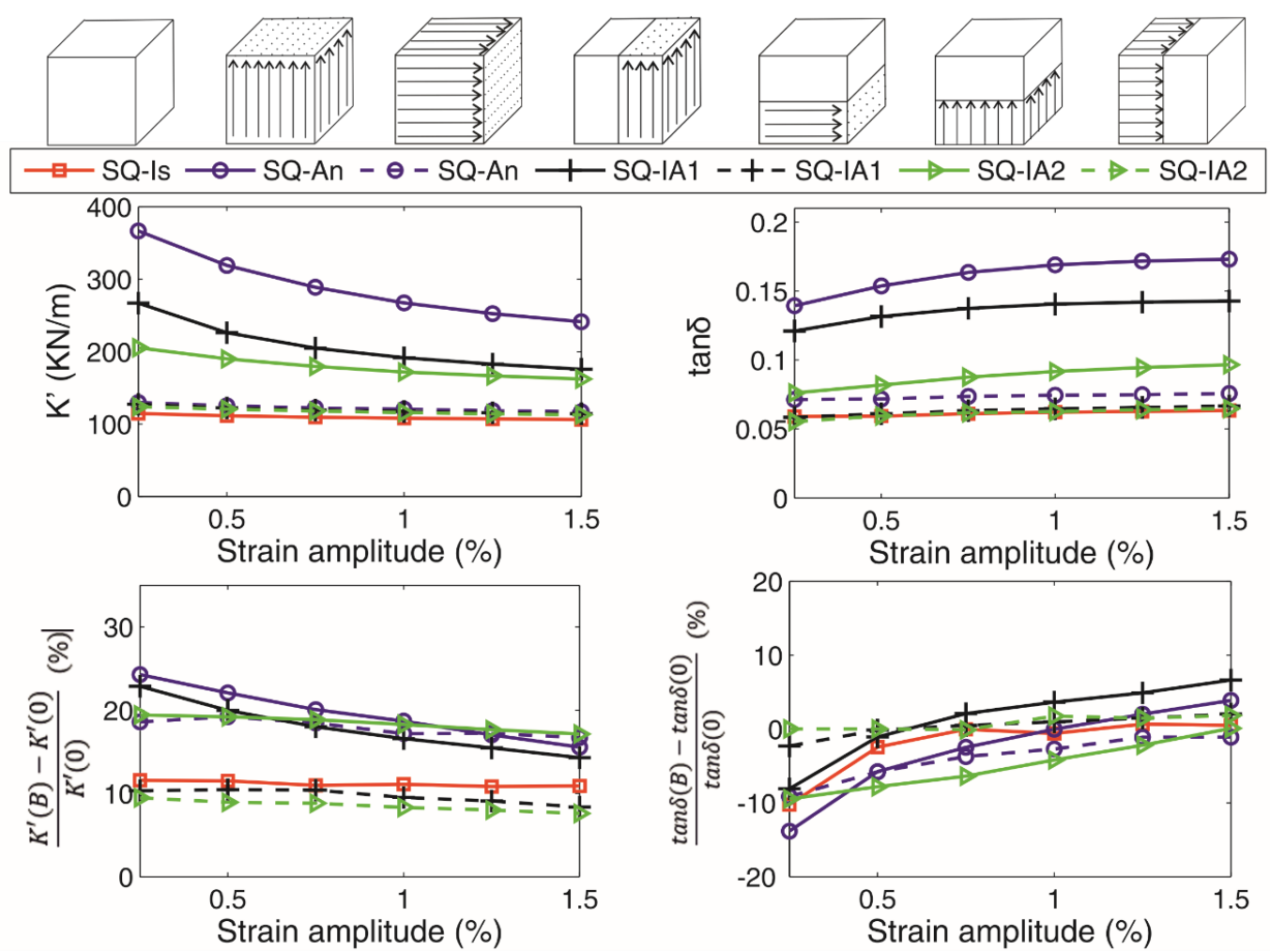

Figure 9: Zero-field values and MR effect of storage E' modulus, loss E"' modulus and tangent of the loss angle $\tan \delta$ of anisotropic SQ-An, isotropic SQ-Is, composite SQ-IA1 and SQ-IA2 MREs in respect to strain amplitude (at $5 \mathrm{~Hz}$ frequency and $10 \%$ prestrain). Solid lines represent axial stiffness while dashed lines the transverse.

Isotropic MRE has the lowest real dynamic stiffness K' MR effect, of about 10\%, that does not vary with strain amplitude while, $\tan \delta$ is not influenced by the magnetic field. The highest MR effect is observed for the axial real dynamic stiffness $\mathrm{K}^{\prime}$ of anisotropic MRE SQ-An that decreases with increasing strain amplitude, dropping from $25 \%$ at $0.25 \%$ amplitude to $18 \%$ at $1.5 \%$ amplitude while MR effect of $\tan \delta$ increases from $-14 \%$ to $+2 \%$. The MR effect of transverse real dynamic stiffness $\mathrm{K}$ ' of anisotropic MRE SQ-An is $8 \%$ higher than isotropic sample that is not affected much by strain amplitude. At strain amplitude values above 1\%, anisotropic sample SQ-An has the same axial and transverse real stiffness K' MR effect.

The relative MR effect values reported here are lower than those mentioned in the previous section for disk samples, due to the lower magnetic field created by the permanent magnets when the distance between them increases from $12.5 \mathrm{~mm}$ to $22 \mathrm{~mm}$. The cube shaped anisotropic sample SQ-An behaves differently from the disk anisotropic sample An-s, where storage modulus E' MR effect increased for 
Sapouna et al, composite MREs

small strain amplitudes to decrease later for strain values above $0.75 \%$. We believe that is due to the different shape and magnetic field while testing and curing, since disk samples were cured under $0.5 \mathrm{~T}$ while cube samples under $0.35 \mathrm{~T}$. Thus, the filler particles-elastomer matrix structure is different for each anisotropic sample that affects how particles can move inside the matrix.

The axial real dynamic stiffness K' and tan $\delta$ MR effects of composite isotropic/anisotropic SQ-IA1 sample are only $2 \%$ less and 5\% more positive respectively than the axial equivalent of anisotropic SQAn sample, but follow the same trend with increasing strain amplitude. The transverse real dynamic stiffness K' and $\tan \delta$ MR effects, of $10 \%$ and $0 \%$ respectively are very close to isotropic sample SQ-Is and are not influenced much by strain amplitude. The axial and transverse real dynamic stiffness $\mathrm{K}$ ' MR effects of composite isotropic/anisotropic SQ-IA1 sample are $20 \%$ and $10 \%$ respectively while both only drop by $2 \%$ when strain amplitude increases from $0.25 \%$ to $1.5 \%$. The axial tan $\delta$ MR effect increases monotonically from $-10 \%$ to $-2 \%$ with increasing strain amplitude, while the transverse $\tan \delta$ MR effect remains zero throughout the strain amplitude range.

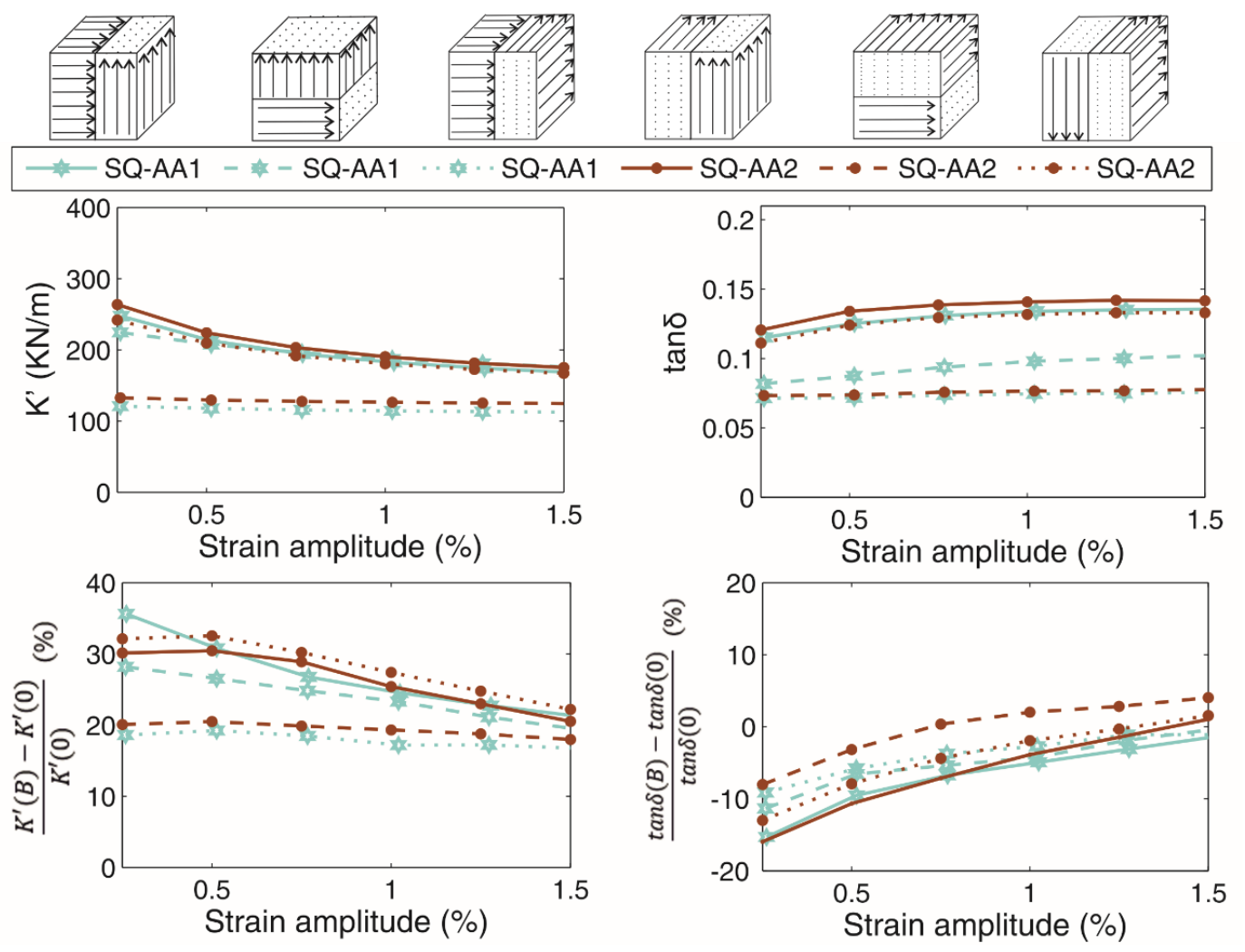

Figure 10: Zero-field values and MR effect of storage E' modulus, loss E" modulus and tangent of the loss angle $\tan \delta$ of composite SQ-AA1 and SQ-AA2 MREs in respect to strain amplitude (at $5 \mathrm{~Hz}$ frequency and $10 \%$ prestrain). Solid lines represent axial stiffness, dashed lines the transverse and doted lines the longitudinal.

The variation of zero field real dynamic stiffness K' and tangent of the loss angle tan $\delta$ and their relative MR effect of composite SQ-AA1 and SQ-AA2 MRE samples in respect to strain amplitude are 
Sapouna et al, composite MREs

illustrated in Figure 10. Sample SQ-AA2 has the same axial and longitudinal real stiffness K' and $\tan \delta$ with the axial K' and tan $\delta$ of samples SQ-AA1 and SQ-IA1, while they follow the same trends with increasing strain amplitude. On the other hand, sample SQ-AA2 has the same transverse real stiffness $\mathrm{K}^{\prime}$ and $\tan \delta$ with the longitudinal $\mathrm{K}^{\prime}$ and $\tan \delta$ of sample SQ-AA1 and transverse $\mathrm{K}^{\prime}$ and $\tan \delta$ of sample SQ-IA1.

The equivalent real stiffness K' MR effect of samples SQ-AA1 and SQ-AA2 is almost 10\% higher than sample SQ-IA1 in all directions. We believe this is due to the interface layer between the two anisotropic parts since the prestructure of the particle allows them to move easier inside the matrix without restricting each other's path like the anisotropic-isotropic interface layer case. However, the equivalent real stiffness K' MR effect of samples SQ-AA1 and SQ-AA2 is even higher than the pure anisotropic SQ-An sample itself, while tan $\delta$ MR effect is close to the one of pure anisotropic sample. This result agrees with the findings of the theoretical study of Allahyarov et al [16] on the magnetoelastic response of bilayered magnetic elastomers, where the composite elastomer responded more efficiently to an external magnetic field than a single component material.

\section{Conclusion}

In this work, we examined the possibility of combining isotropic and anisotropic MREs in parallel and series configurations to a novel composite MRE as an easy way to tailor its dynamic mechanical properties. The new composite MRE has higher tangent of the loss angle tan $\delta$ than isotropic MRE by keeping stiffness lower than anisotropic MRE without compromising the MR effect. Under a $0.5 \mathrm{~T}$ magnetic field, pure anisotropic and isotropic MREs increased their storage modulus E' by $31 \%$ and $21 \%$ respectively while their parallel and series combination composite MREs by $27 \%$ and $28 \%$ respectively.

The experimental results showed that the pure isotropic MRE made of large (diameter $<220 \mu \mathrm{m}$ ) particles has similar zero field dynamic properties but a slightly lower MR effect than the isotropic MRE made of small (diameter 4-6 $\mu \mathrm{m}$ ) particles. The anisotropic MRE with large particles is slightly stiffer but have higher damping than the equivalent sample with small particles but their MR effect is $10 \%$ lower. In addition, a strong MR effect-strain amplitude coupling effect was observed for all samples except anisotropic MREs with large particles that tend to form more stable matrix-particles structures.

The exact stiffness and damping capability of composite samples can be tailored by selecting the dimensions of each part and the particle size that each MRE part is made of. For example, using an anisotropic MRE core made of large particles to a cylinder isotropic MRE made of small particles, increases tangent of the loss angle for small strains by $25 \%$ while keeping stiffness the same with the case where the anisotropic core is made of small particles. However, there is a 5\% compromise on the MR effect. 
Sapouna et al, composite MREs

Finally, combining two anisotropic MREs with particles aligned in two different directions produces a MRE with similar zero field dynamic mechanical properties and MR effect on those directions. Under a 0.35T magnetic field these anisotropic/anisotropic composite MREs increase their dynamic stiffness by $32 \%$ which is $10 \%$ higher than the equivalent pure anisotropic MRE. This is highly desirable in applications where the elastomer is under multidirectional loading conditions. The length of each part can be selected to create a MRE with the desired axial and transverse stiffness and damping, having in mind that a compromise between them will always exist. In the future, we aim to test these novel composite MREs in a single degree of system mass-MRE inclined isolator where the elastomer will be subjected to both shear and compression loading conditions.

\section{References}

[1] Schubert G and Harrison P 2016 Equi-biaxial tension tests on magnetorheological elastomers Smart Mater. Struct. 25015015.

[2] Khimi S R and Pickering K L 2015 Comparison of dynamic properties of magnetorheological elastomers with existing antivibration rubbers Composites Part B 83 175-183.

[3] Schubert G, Harrison P 2015 Large-strain behaviour of Magneto-rheological Elastomers tested under uniaxial compression and tension, and pure shear deformations Polymer Testing 42 122134

[4] Kallio M, Lindroos T, Aalto S, Järvinen E, Kärnä T and Meinander T 2007 Dynamic compression testing of a tunable spring element consisting of a magnetorheological elastomer Smart Mater. Struc. 16 269-278

[5] Wei B, Gong X and Jiang W 2010 Influence of polyurethane properties on mechanical performances of magnetorheological elastomers Journal of Applied Polymer Science 116 771778

[6] Mitsumata T, and Ohori S 2011 Magnetic polyurethane elastomers with wide range modulation of elasticity Polym.Chem. 21063

[7] Hu Y, Wang YL, Gong X L, Gong X Q, Zhang X Z, Jiang W Q, Zhang P Q and Chen Z Y. 2005 New magnetorheological elastomers based on polyurethane/Si-rubber hybrid Polymer Testing 24 (3): 324-329.

[8] Li R, and Sun L Z. 2013 Viscoelastic responses of Silicone-Rubber-Based Magnetorheological Elastomers Under Compressive and Shear Loadings Journal of engineering Materials and Technology 135: 021008-1 -7.

[9] Yang J, Gong X G, Deng H, Qin L and Xuan S 2012 Investigation on the mechanism of damping behavior of magnetorheological elastomers Smart Mater. Struct. 21125015 
Sapouna et al, composite MREs

[10] Bose H, Roder R 2009 Magnetorheological elastomer with high variability of their mechanical properties 11th Conference on Electrorheological Fluids and Magnetorheological Suspensions J. Phys.:Conf. Ser. 012090

[11] Popp KM, Kroger M, Li WH, Zhang XZ and Kosasih PB 2010 MRE Properties under shear and squeeze modes and applications J Intell Mater Syst Struct. 21 1471-1477

[12] Borbath T, Gunther S, Borin D Yu, Gundermann Th and Odenbach S 2012 XmCT analysis of magnetic field induced phase transitions in magnetorheological elstomers Smart. Mater. Struct. 21105018

[13] Gordaninejad F, Wang X and Mysore P. 2012 Behavior of thick magnetorheological elastomers Intelligent Material Systems and Structures 23 (9): 1033-1039.

[14] Li Y, Li J, Li W and Samali B 2013 Development and characterization of a magnetorheological elastomer based adaptive seismic isolator Smart Mater. Struct. 22035005

[15] Snowdon J 1968 Vibration and shock in damped mechanical systems (New York: Wiley)

[16] Allahyarov E, Menzel A M, Zhu L and Lowen H 2015 Magnetomechanical response of bilayered magnetic elastomers Smart Mater. Struct. 23115004

[17] Xin F L, Bai X X and Qian L J 2016 Modeling and experimental verification of frequency-, amplitude-, and magnetodependent viscoelasticity of magnetorheological elastomers Smart Mater. Struct. 25105002

[18] Sorokin V V, Ecker E, Stepanov G V, Shamonin M, Monkman G, Kramarenko E Yu and Khokhlov A R 2014 Experimental study of the magnetic field enhanced Payne effect in magnetorheological elastomers Soft Matter 10 8765-8776.

[19] Davey A B, and Payne A R 1965 Rubber in Engineering Practice (London: Maclaren \& Sons LTD)

[20] Yang J, Gong X G, Deng H, Qin L and Xuan S 2012 Investigation on the mechanism of damping behavior of magnetorheological elastomers Smart Mater. Struct. 21125015 